\title{
eHealth WBAN: Energy-Efficient and Priority-Based Enhanced IEEE802.15.6 CSMA/CA MAC Protocol
}

\author{
Wireless Body Area Network
}

\author{
Ansar Munir Shah ${ }^{1}$, Abdelzahir Abdelmaboud $^{2}$, Khalid Mahmood $^{3}$, \\ Mahmood ul Hassan ${ }^{4}$, Muhammad Kashif Saeed ${ }^{5}$ \\ Department of Computer Science \\ IIC University of Technology \\ Phnom Penh, Cambodia
}

\begin{abstract}
This paper provided a general study of Wireless Body Area Network (WBAN) in health monitoring system as well as the study of the application of wearable and implanted BioMedical-Sensors (BMS) which are used to monitor the vital signs of a patient in early detection. Energy efficiency is a significant issue in WBAN which can be achieved by reducing the overhead of control packets, prioritizing sensor-nodes and sink-node selection. Moreover, uncertainty in network topologies, such as distance and link affect between sensor-nodes occurs due to the mobility of human. In this research, we propose a scheme to reduce the overhead of control packets and prioritizing the threshold values of vital signs by assigning low and high transmission power with enhanced IEEE802.15.6 CSMA/CA as well as introduce a Mobility Link Table (MLT) for selecting a sink-node to communicate with the coordinator. Compare it with existing IEEE802.15.6 CSMA/CA technique and results shows the proposed techniques regarding mean power consumption, network delay, network throughput.
\end{abstract}

Keywords-Wireless Body Area Network (WBAN); node; IEEE802.15.6; MAC; CSMA/CA; Mobility Link Table (MLT)

\section{INTRODUCTION}

A WSN is a manner to organize sensors nodes to observe and control the environmental factors such as temperature, motion detection, sound, and send their data to the base station [1]. WSN consists of various applications in the fields of medical, military and supports [2]. The use of wireless sensors in the medical field is considered as most emerging and evolving wireless network to monitor the patient anytime anywhere is known as WBAN wireless body area network. A Wireless Body Area Network (WBAN) is special-purpose sensor network which is designed for medical applications and operates independently to control communication between various medical sensors, which are to be placed inside and outside the human body Patient with long-standing diseases required continuous supervision. With restrict resources, it is almost impossible to focus on every patient continuously in and outside the hospital, so it is need of the time for healthcare services to become more efficient to monitor and examine the patient anytime and anywhere [3].

Sensors used in medical monitoring are tiny, so WBAN is required many parameters such as low power consumptions, low latency, reliability, efficient bandwidth utilization and maximum throughput. The batteries used in these types of sensors are also tiny, and it is challenging to recharge or replace them, so low power consumption is considered as a significant challenge for effective WBAN. Therefore energy efficient MAC protocol is needed to overcome this challenge [4]. WBAN can be made energy efficient by updating in MAC layer as well as the power control mechanism, so reducing the overhead of control packets, prioritizing nodes and efficient sink selection using one-hop topology are concerning areas to save the energy in WBAN [5]. The patients change their postures according to their needs and posture mobility of patient body effects distance and connections variation between different sensor nodes.

In this research, we will propose two different protocol techniques. First technique will rely upon reducing the overhead of control packets by using block acknowledgment for data packets and prioritizing nodes according to the transmission power and contention window size. Second technique will relate to adaptive sink selection, which permits sink selection by using Mobility Link Table (MLT). The simulated analysis describes that purposed mechanism is succeeded to reduce mean power consumption in WBAN.

In Section II, existing media access control mechanism is described. Section III contains the proposed schemes and experimental setup and analysis are evaluated in Section IV, as well as the Section V concludes the final recommendation and future work.

\section{EXISTING MEDIA ACCESS CONTROL MECHANISMS}

MAC frame consists of MAC header, payload, and FCS; payload holds the informative data, and MAC header hold Control Bit frame, Recipient-identity, Sender-identity and BAN-identity [6]. Usually, B-MAC, L-MAC, S-MAC and Wise MAC protocols are used in WBAN.

B-MAC (Berkeley Mac protocol) [7], low power listening protocol, have to wake up and sleep states of the nodes which described that of B-MAC is to make nodes sleepy for a long time interval and to wake up the nodes after fixed intervals to check ongoing data communication. Timeout- timer stop the data listing if it does not find any data packet for that time, else the node will wait for entire data packets. The sender node announces a preamble to other nodes before sending the data 
packet B-MAC is an asynchronous protocol and fairness is not guarantee in B-MAC. CCA (Clear Channel Assessment) is a key ingredient in B-MAC and CCA mean to detect clear channel for data or control packet transmission. Data receiving node will send an Acknowledgement packet to data sending node, but it is optional, and no priority mechanism used to assign the slot to the emergency data in a critical situation and also does not considered the threshold values of vital signs simultaneously.

Lightweight Medium Access (LMAC) protocol is TDMAbased protocol [8]. Slot assignment and synchronization in an LMAC network is self-organizing. This protocol reduces energy by avoiding a collision, minimizing overheads on the physical layer, and by reducing the state-switching in the transceiver when changing the sleep-interval for nodes according to the amount of data traffic. In LMAC [9], collision is reduced by dividing time into multiple slots and assigned specific time-slot to each node for a specific time, So that each node can send its data in allocated time-slot and don't need to compete for medium. A distributed algorithm used for time slot assignment. Both sender and receiver will turn off their transceivers after data transmission and reception respectively for saving the energy consumption. The node synchronization process is significant for LMAC, achieved by gateway control messages. Each node, on reception of gateway message, will synchronize their clocks with a gateway of their one-hop neighbor, on the other hand, it does not handle the thresh hold values of vital signs at the same time, and fixed slot allocation caused delay.

Sensor-MAC protocol considered energy-efficient media access control protocol. According to [1], SMAC reduces energy by avoiding a collision, control packet overhead, overhearing, idle listening. LMAC recognizes sleep and listens to phases for transceiver node so there should be synchronization between the network-nodes for sleeping and listen, but synchronization is not strict sometime between the nodes so they can change their sleep period as they required communication need [9]. Periodic sleeping is an essential component of SMAC which means node will change its state to sleep state in the absence of data transmission or reception. During sleep period, the node controls its wireless off, and awake it-self later according to the timer. Both contention and scheduling techniques are deployed in this protocol to reduce data collision. Fairness is considered in protocol transmission but the delay increased.

Wise-MAC is known as high energy-efficient MAC protocol presented in [10]. Wise-mac is based upon nondeterministic CSMA and became energy-efficient by using preamble sampling, and by reducing idle-listening. Idlelistening reduced by listening medium for short interval of time. The process of listening will continue until data-packet is received or medium becomes idle until finding the channel busy. Many nodes are used as relays towards the sink to avoid collision between data packets and further collision can be reduced by using medium reservation preamble in the wake-up preamble. If long data packet transmission is required, then an extra bit called more is added in front of the data packet to indicate receiver to listen, even after sending an acknowledgment. The sender sends the second packet ongoing to the received ACK-packet. CSMA/CA protocol is based on "contention-based technique". This MAC frame structure is shown in Fig. 1.

The whole channel is divided into super-frame hierarchy [11]. In each beacon period, beacons are broadcast. In the inactive period, no beacon is transmitted. Fig. 1 represents the Beacon mode with beacon super-frame edges, Fig. 2 and 3 shows the Non-Beacon mode with super-frame edges and without super-frame edges, respectively.

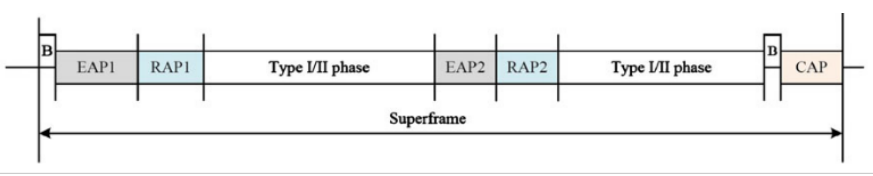

Fig. 1. Beacon mode with beacon super-frame edges.

In CSMA/CA the node detects the channel, and if find it idle, the node will broadcast the data, and if find it busy, the node will wait for random time and will try again. The node sets a back-off counter between 1 and contention window (CW) size. Node senses the random channel time, and if the channel idle, the counter decrement one at every idle and transmit the frame on the channel if the back off counter becomes zero. If the channel is found busy, the node will lock its back-off counter until the channel becomes idle. A number of failures occur are counted by another counter [12]. There are two cases possible to represent the failure solution, the Contention window (CW) size will remain unchanged if failures counter recorded odd value, the $\mathrm{CW}$ will be doubled if failure counter value noticed even. After data transmission completed, contention window-size is set to initial CW.

IEEE 802.15.6 based CSMA/CA [13] describes the MAC protocol with immediate acknowledgment and without acknowledgment on same contention window size (without user priority) and calculate the network delay, throughput and bandwidth efficiency. This scheme is implemented only for one sender, and one receiver node, multi-nodes, and postural mobility is not considered in the simulation. While in WBAN, we need a mobile network along with more than one receiver and transmitter.

\section{PROPOSED SCHEMES}

Propose technique aims to improve the overall network throughput and reduce the mean power consumption considering body mobility and posture variation factors in Intra-WBAN and evaluate the performance metrics of mobility network. Eleven nodes in the network and introduce mobility parameters to evaluate the performance parameter of the network. For energy efficiency in WBAN, we prioritize nodes by critical/emergency traffic and non-critical/normal traffic. Normal and emergency traffic are differentiated by using two techniques, one by different contention window (CW) sizes and another one is by using different transmission power for the different type of traffic. In IEEE 802.15.6 based CSMA/CA protocol, only immediate acknowledgment policy is implemented. In proposed protocol, we use block acknowledgment policy which reduces the control packet overhead so that we can get efficient power consumption in WBAN. 
In the first scheme, we proposed a superframe with RAP1 and RAP2 with prioritizing the nodes by using different transmission power and different contention window size for each node. The sensor node with higher transmission power, let's call P1, will get more priority, and higher power will be given to node generating emergency traffic while node generating normal traffic will be given lower power let's say P2 which will effectively decrease the priority of that node as compared to the node with emergency traffic. Due to the high priority of emergency traffic generating node, its traffic will reach the destination without distortion by the collision which will produce less delay. Proposed technique (enhancement of IEEE 802.16.4) packet collision scenarios are defined in two states, as shown in Fig. 2, i) S1: More than one low powered packets arrival at t2 ii) S2: More than one High powered packets arrival at t5. In scenario 1 , more than one low priority packets arrive which causes a collision between them. The packet collision cause discard of the packet and no packet received at the destination. Similarly, in scenario 2, there is more than one high priority packet arrived which also results in a collision.

A packet without collision scenarios are defined in three states, as shown in Fig. 3, i) S1: one high powered packet arrival, ii) S2: one low powered packet arrival, iii) S3: one high powered packet and more than one low powered packet arrival. When one high or one low priority packet arrives, it is received at the destination without collision. In scenario 3, one high powered and one low powered packet is received, as per our policy, the high powered packet will get priority and will reach the destination without collision.
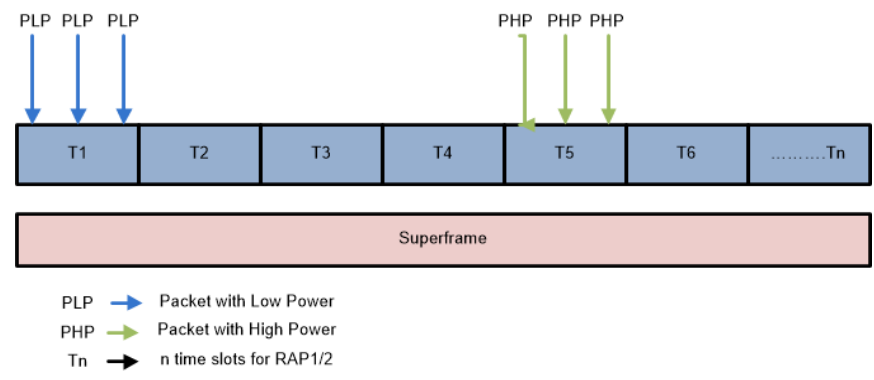

Fig. 2. Collided packet transmission with Enhanced IEEE 802.15.6.

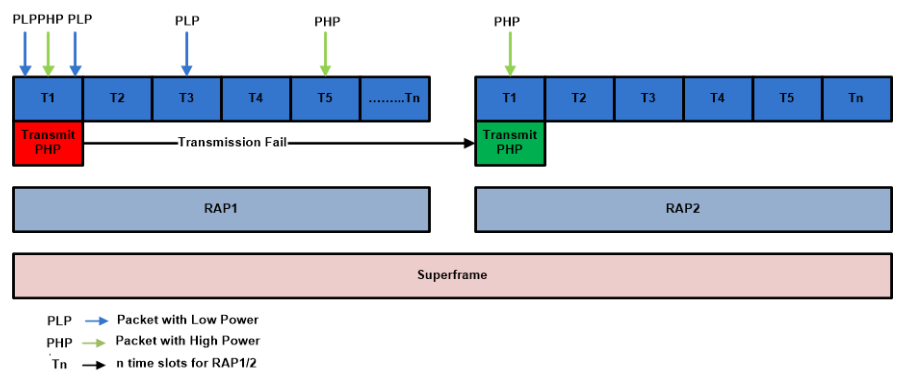

Fig. 3. Successful packet transmission in Enhanced IEEE 802.15.6.
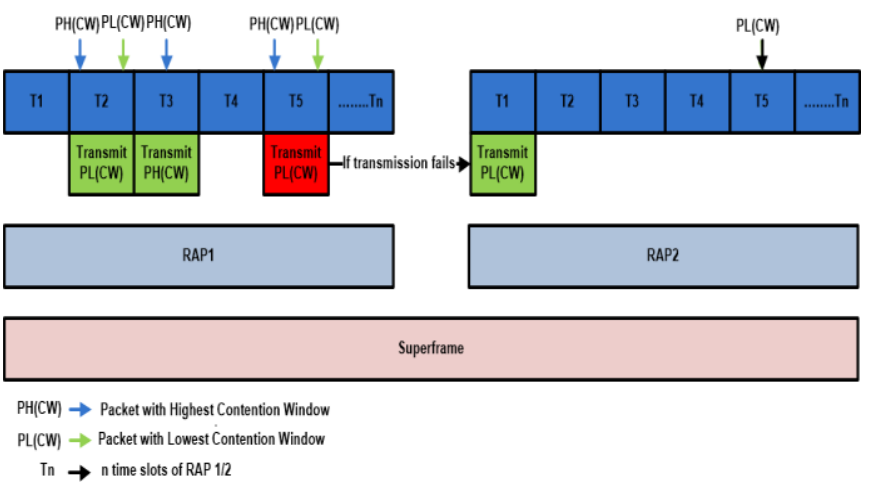

Fig. 4. Priority using contention window size.

The second method to assign user priority is by using different contention window size for each node. A node with minimum contention window size will get higher priority while a node with maximum contention window size will get lower priority. Emergency traffic will get the highest priority, so it will access the channel first due to which optimal delay and throughput are obtained. In Fig. 4 the access mechanism of the the channel is illustrated, which has three cases: i) Only low priority node transmit data ii) Only high priority node transmit data ii) High and low priority node transmit data. In case 1, at time T2 there are two packets, one with high contention window and another one with low contention window trying to access channel. The packet with low contention window will access the channel firstly, while the high window and another one with low contention window trying to access channel. The packet with low contention window will access the channel firstly, while the high contention window sized packet will wait for its turn. While in scenario 2 and 3 at time T4 and T6, one low contention window sized packet and one high contention window sized packet are trying to access the channel, so they will send their packets through the channel without collision which will ultimately cause minimum delay.

The second scheme introduced a mechanism to choose a sink node to communicate with the coordinator in IntraWBAN. In WBAN, it is imperative to make the data transmission easy by choosing the easily accessible sink node for all other sensor nodes. In Intra-WBAN, distance and connection between different nodes vary due to the variation in posture or mobility in the human body, as shown in Fig. 5. Energy waste is a significant problem in WBAN due to improper sink selection technique and the maximized control overhead [14]. Using fix-sink or random-sink selection, using the routing table are the conventional techniques in WBAN. However, we know that information collected from every node is critical for the doctor to take any decision for patient treatment, so we need active techniques in which all nodes are accessible by the sink. Because in previous techniques other nodes may or may not access that the sink does not access particular node or other nosed. 

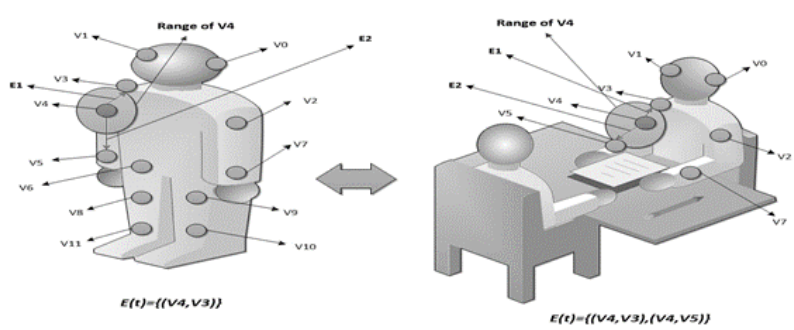

Fig. 5. Illustration of range changing of sensor node as posture changes.

As we are considering the human mobility, the MLT is a significant feature of our technique. This table contains information about no. of connections; each node has a variable called "Link" which will be incremented after each connection between two nodes is verified, through acknowledgment. MLT will use this variable to store a number of connections by each node. An MLT is distributed and maintained by each node. Complete procedure to fill this table and selecting adaptive sink by MLT described in Fig. 6.

According to Fig 6, each sensor node will broadcast control packet. All the nodes reply after getting the control packet from another node, MLT will be updated (Incrementing No. of Links) if ACK received. A node with maximum "link" value in MLT presents the sink node in selected posture. Table update before every data transmission against the current posture and node that have more links will be select as a sink node. Sink will be updated before transmission by repeating the same procedure. In this paper, we compare proposed sink selection with fixed sink, random sink selection through routing table.

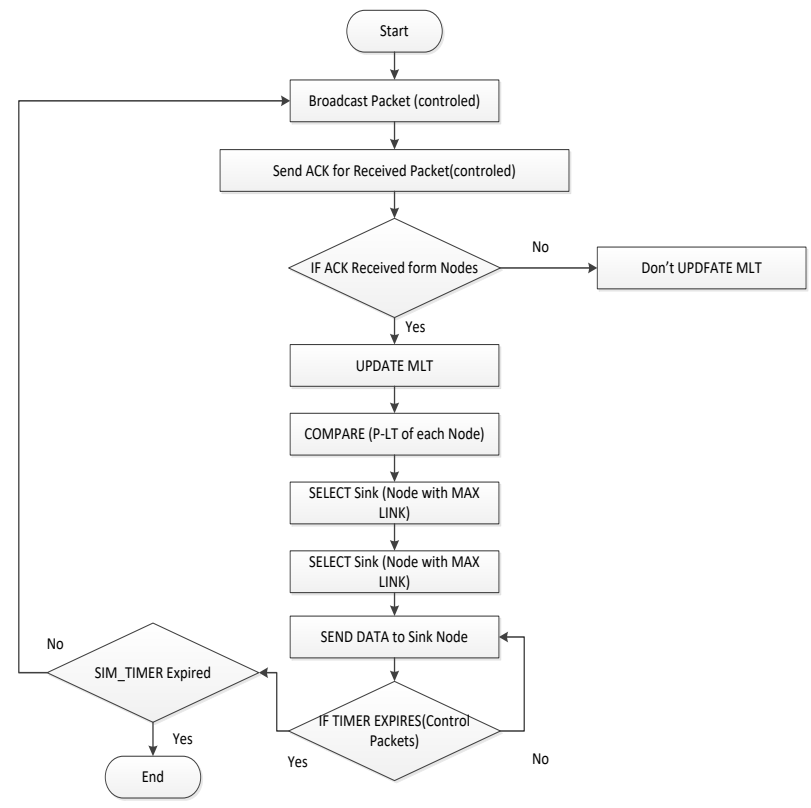

Fig. 6. Flow chart for adaptive sink selection by MLT.

\section{EXPERIMENTAL SETUP}

Proposed scheme are compared with IEEE802.15.6 CSMA/CA by simulated in OMNET++/MIXIM. Fig. 7(a) shows network delay comparison between proposed MAC and IEEE 802.15.6 based CSMA/CA by prioritizing using Contention Widow (CW) mechanism as well as the power priority mechanism. Fig. 7(b) shows network bandwidth efficiency comparison with IEEE 802.15.6 based CSMA/CA using $\mathrm{CW}$ and also prioritizing the nodes by the transmission power.
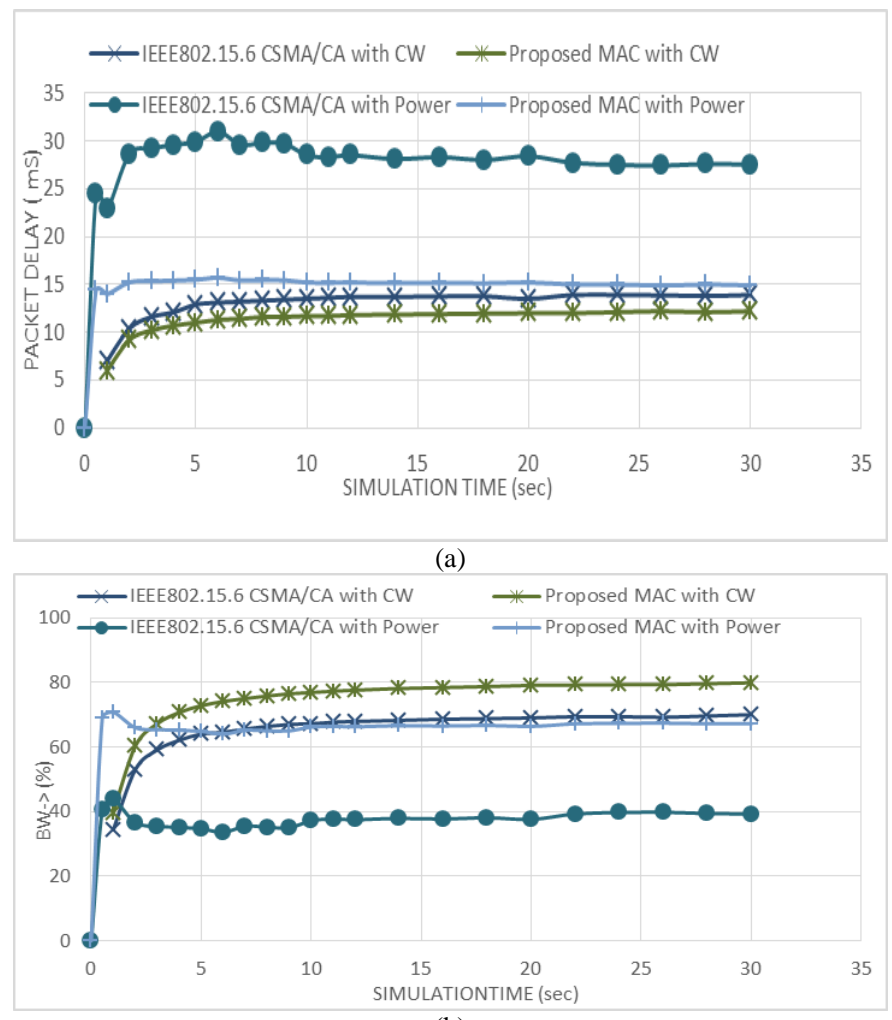

(b)

Fig. 7. (a) Network delay, (b) Network bandwidth.

Fig. 7(a) \& (b) shows that the minimum network delay, maximum network throughput, and maximum bandwidth efficiency is also achieved for the network, in proposed MAC prioritizing by contention window $(\mathrm{CW})$ due to less control packet overhead and nodal prioritization. As depicted from this figure that highest network delay, lowest throughput, and lowest bandwidth efficiency is present in case of IEEE 802.15.6 based CSMA/CA with CW. The proposed MAC with priority using contention window $(\mathrm{CW})$ is showing optimum results because node with the highest priority uses the channel for a maximum time because that node has maximum data for transmission and need a channel for maximum time. Fig. 8 shows that minimum power consumption is achieved in proposed MAC by prioritizing using transmission power with block acknowledgment because when we use different transmission power and give the highest priority to highest transmission power node.

We know that nodes with normal traffic will transmit data using lower transmission power because we have assigned less priority to normal traffic. Total power consumption is the sum of transmission power, receiving power and power used in acknowledgment. Moreover, transmission power has the highest influence on power consumption, as normal traffic is mostly used, so transmission power of the whole network will be reduced which means that average transmission power is reduced so that we will get less power consumption. Moreover, 
block acknowledgment case further improves results by decreasing control packet overhead, which further reduces power consumption.

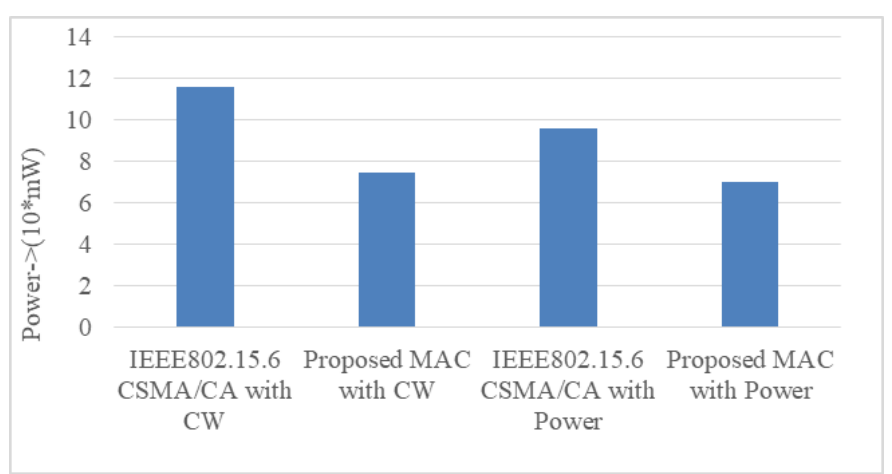

Fig. 8. Network mean power consumption.

Fig. 9 shows power consumption comparison between fixed sink, random sink selection technique, and adaptive sink selection technique through MLT, respectively for the network.

The performance analysis shows lower delay, lower power consumption, higher throughput, and higher bandwidth efficiency is achieved in adaptive sink selection technique through MLT. In adaptive sink-selection, a sink-node has maximum LINK available technique results more comfortable accessible for other nodes, which improve the performance results. On the other hand, in random sink-selection approach, selected sink-node may or may not have enough amount of connections cause more delay, high energy consumption and lower throughput.

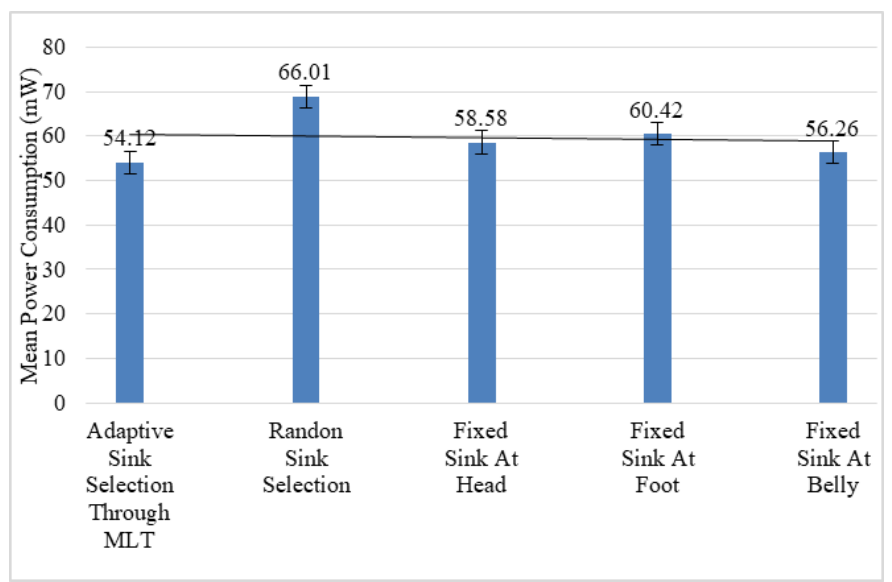

Fig. 9. Network power consumption on different sink.

\section{CONCLUSION}

Increased control packet overhead and inappropriate sink selection techniques are considered as energy wastage factor in Intra-WBAN. Two techniques proposed in this paper, the first technique helps in reducing control packet overhead, and its prioritization feature gives priority to nodes according to the power and contention window size. The second technique is based upon adaptive sink selection through MLT. These two schemes help in reducing network delay, mean power consumption, and increasing network throughput. In the first technique, control packet overhead is reduced by sending acknowledgment after 5 data packets. A second key feature which is present in this technique is two ways of assigning nodal priority according to the transmission power and contention window size, where transmission power and contention window size of every node is unique.

In the first case, the node with maximum transmission power will be assigned highest priority and priority will decrease as transmission power reduces. In the second case, priority will increase as $\mathrm{CW}$ size reduces and so on. In the second technique, sink node for data packet will be adaptive to the variation in a number of connections that each node holds. A number of connections for every node are present in MLT. A node with a maximum number of connections will be made sink node, and other nodes will send data to this node.

Performance analysis shows that proposed techniques outperform then IEEE 802.15.6 based CSMA/CA considering different evaluation metric as presented in performance analysis metrics such as mean power consumption, network delay, network throughput and network bandwidth efficiency. Results show that our proposed techniques are showing ten to fifteen percent improvement in IEEE 802.15.6 based CSMA/CA. We want to further enhance our work by introducing the sink node selection by links and energy power maintain by sensor nodes considering the human mobility and posture variation in Intra-WBAN. As a test - the bed is provided in OMNET++ $\backslash$ MIXIM that is why other researchers can also enhance our work.

\section{ACKNOWLEDGMENT}

We would like to express our gratitude to IIC University of Technology, the Kingdom of Cambodia for providing us excellent research environment.

\section{REFERENCES}

[1] Ye, Wei, John Heidemann, and Deborah Estrin. "An energy-efficient MAC protocol for wireless sensor networks." In INFOCOM 2002. Twenty-First Annual Joint Conference of the IEEE Computer and Communications Societies. Proceedings. IEEE, vol. 3, pp. 1567-1576. IEEE, 2002.

[2] Park, KeeHyun. "A Ubiquitous Motion Tracking System Using Sensors in a Personal Health Device." International Journal of Distributed Sensor Networks2013 (2013).

[3] Khan, Jamil Yusuf, Mehmet R. Yuce, Garrick Bulger, and Benjamin Harding. "Wireless body area network (WBAN) design techniques and performance evaluation." Journal of medical systems 36, no. 3 (2012): 1441-1457.

[4] Cavallari, Riccardo, et al. "A survey on wireless body area networks: Technologies and design challenges." IEEE Communications Surveys \& Tutorials 16.3 (2014): 1635-1657.

[5] Mehmood, Amir, and Adnan Nadeem. "Self-Organized Routing in Body Area Sensors Networks: A Review, Analysis and Simulation-based Case Study." Pakistan Journal of Engineering and Applied Sciences (2016).

[6] Kwak, Kyung Sup, Sana Ullah, and Niamat Ullah. "An overview of IEEE 802.15. 6 standard." Applied Sciences in Biomedical and Communication Technologies (ISABEL), 2010 3rd International Symposium on. IEEE, 2010.

[7] Forster, A. "Implementation of the B-MAC Protocol for WSN in MiXiM."Networking Laboratory, University of Applied Sciences of Southern Switzerland(2009).

[8] Ali, Mahmood, Annette Böhm, and Magnus Jonsson. "Wireless sensor networks for surveillance applications-a comparative survey of mac protocols." Wireless and Mobile Communications, 2008. ICWMC'08. The Fourth International Conference on. IEEE, 2008. 
[9] Van Hoesel, Lodewijk FW, and Paul JM Havinga. "A lightweight medium access protocol (LMAC) for wireless sensor networks: Reducing preamble transmissions and transceiver state switches." 1st International Workshop on Networked Sensing Systems (INSS. Society of Instrument and Control Engineers (SICE), 2004.

[10] El-Hoiydi, Amre, and Jean-Dominique Decotignie. "WiseMAC: An ultra low power MAC protocol for multi-hop wireless sensor networks." Algorithmic Aspects of Wireless Sensor Networks. Springer Berlin Heidelberg, 2004. 18-31.

[11] Crosby, Garth V., et al. "Wireless body area networks for healthcare: A survey." International Journal of Ad Hoc, Sensor \& Ubiquitous Computing 3.3 (2012): 1.
[12] Ullah, Sana, Min Chen, and Kyung Sup Kwak. "Throughput and delay analysis of IEEE 802.15. 6-based CSMA/CA protocol." Journal of medical systems 36.6 (2012): 3875-3891.

[13] Ait Zaouiat, C. E., and A. Latif. "Performances Comparison of IEEE 802.15. 6 and IEEE 802.15. 4." INTERNATIONAL JOURNAL OF ADVANCED COMPUTER SCIENCE AND APPLICATIONS 8.11 (2017): 461-467.

[14] Baig, Muhammad Rahim, et al. "AnyCasting In Dual Sink Approach (ACIDS) for WBASNs." INTERNATIONAL JOURNAL OF ADVANCED COMPUTER SCIENCE AND APPLICATIONS 8.3 (2017): 257-263. 